\title{
CHANGES IN LEISURE-TIME PHYSICAL ACTIVITY LEVELS AND PERCEIVED BARRIERS AMONG ESTONIAN ADULTS OVER A TWO-YEAR PERIOD
}

\author{
PEETER LUSMÄGI ${ }^{1}$, KERLI MOOSES ${ }^{2}$, EVE-LIIS ROOSMAA ${ }^{1}$, \\ MERIKE KULL ${ }^{2}$ \\ ${ }^{1}$ Institute for International Social Studies, School of Governance, \\ Law and Society, Tallinn University, Tallinn, Estonia \\ ${ }^{2}$ Institute of Sport Sciences and Physiotherapy, Faculty of Medicine, \\ University of Tartu, Tartu, Estonia
}

\begin{abstract}
The aim of this study is to describe the changes in leisure-time physical activity (LTPA) levels among the Estonian adult population in the years 2013-2015 in order to examine the relationships between LTPA level and socioeconomic status (SES) and health indicators, and also to explore perceived barriers to LTPA. Data from the National Physical Activity Survey were used. The sample consisted of 1009 participants aged 15-69 in 2013 and 1004 respondents in 2015. The proportion of Estonian adults exercising regularly at least four times per week increased from 39\% in 2013 to $45 \%$ in 2015 . Being in the older age groups increased the odds for physical inactivity 2.07-4.74 times compared to the youngest age group (15-24) $(\mathrm{p}<0.01)$. Having primary, basic, or secondary education increased the odds of being inactive 2.33-3.14 times compared to respondents with higher education $(\mathrm{p}<0.01)$. The most prevalent barriers to physical activity reported by inactive adults were: 1 ) the absence of interest or unwillingness to make an effort, 2) tiredness at work, and 3) lack of time. In conclusion, despite a slight positive trend, LTPA levels are low. As the perceived barriers depend on age, public health strategies should involve age-specific solutions, especially for older adults.
\end{abstract}

Keywords: leisure-time physical activity, national survey, sociodemographic groups, barriers, Estonia 


\section{INTRODUCTION}

Unhealthy environments and behaviours are the result of the three global trends: globalization, population-ageing, and rapid unplanned urbanization [59]. Physical activity, including sports, is considered to be an important component of a healthy lifestyle [4]. Adults who are physically more active are less likely to develop many chronic diseases compared to adults who are physically less active [33]. In addition, regular exercise and physical activity help to maintain and improve mental and physical health in various adult age groups [14, 35, 37, 42, 59].

Physical activity is defined as any bodily movement produced by skeletal muscles that results in energy expenditure [9]. Physical inactivity has been proven to be the fourth highest risk factor for global mortality, causing an estimated 3.2 million deaths globally [58]. Physical inactivity is one of the main health challenges in developed countries and can be described as a worldwide epidemic [11]. According to the recommendations [59], adults aged 18-64 should do at least 150 min of moderate-intensity aerobic physical activity per week or at least $75 \mathrm{~min}$ of vigorous-intensity aerobic physical activity per week. These two can also be combined. Aerobic activity should be performed at intervals of at least $10 \mathrm{~min}$ [59]. In order to improve physical condition, avoid weight gain, and reduce the risk of chronic diseases, at least the minimum recommended level of physical activity should be achieved [10].

According to a Eurobarometer survey conducted in 28 EU Member States, 59\% of respondents aged 15 and older practice some form of exercise less than once a week or do not exercise at all [19]. People in the Nordic countries are the most physically active whereas people in Southern Europe are the least physically active. The proportion of adults exercising or playing sports at least once per week is $70 \%$ in Sweden, 68\% in Denmark, and 66\% in Finland. However, the greatest proportion of people who responded that they never exercise or play sports can be found in Bulgaria (78\%), Malta (75\%), and Portugal (64\%). Among the Baltic States, the proportion of adults who never exercise or play sport was 36\% in Estonia, 39\% in Latvia, and 46\% in Lithuania [19]. Surveys conducted in Estonia in 2013 and 2014 showed that the proportion of people who engage in leisure-time physical activity at least two times per week for a minimum 30 minutes ranges between $35.8 \%$ and $38.3 \%[32,50]$.

Previous studies have shown a strong correlation between the implementation of recommendations for physical activity and body mass index (physical inactivity is one of the risk factors regarding metabolic health) [46, 49]. In Estonia more than half of the adult population is classified as overweight 
(body mass index above $25.0 \mathrm{~kg} / \mathrm{m}^{2}$ ) and an increase in this proportion has been particularly noticeable during the last decade. According to studies, in 2004 excessive weight was identified in $45.7 \%$ of the male and $42.8 \%$ of the female population [17], whereas in 2016 it was $59.9 \%$ and $51.7 \%$, respectively [51]. It has been advised that public recommendations being developed for combating excessive weight and obesity should focus on both promoting physical activity and decreasing the desire to participate in sedentary activities [47]. Moreover, the physical activity of the population should regularly be monitored, and it is important to pay attention to the differences between subgroups in order to plan and implement strategies promoting physical activity in the population overall.

Physical activity is a physical and dynamic process. Previous research has established that correlates of physical activity could be classified as either: (a) demographic and biological factors, (b) psychological, cognitive, and emotional factors, (c) behavioural attributes and skills, (d) social and cultural factors, (e) physical environment factors, and (f) physical activity characteristics [52]. Previous studies have shown that differences with regard to gender, age, and level of education have been detected [31, 45]. Participation in physical activity is higher among men than women $[22,29,52]$ and physical activity decreases with age $[23,54]$. People with a higher level of education are more likely to participate in leisure-time physical activity than those with a lower level of education [52]. However, there are studies offering contradictory findings concerning age groups and gender. For example, Scheerder et al. [44] found the smallest gender differences in physical activity in Flanders, France, the Netherlands, and Switzerland (remaining under 3\%), whereas Denmark stood out from other European countries with its proportion of physically active women being higher than men. The study carried out in England showed that both men and women in the age group of 50-64 reported their physical activity as more active than the respondents aged 19-24 years, which is different from other countries in Europe [40].

In order to develop physical activity policies and intervention programs, it is important to identify factors that can influence the physical behaviour of adults. One approach is the identification of psychological factors such as knowledge, attitudes, and perceived motivators and barriers in terms of physical activity in different population groups in order to promote effective behaviour change programs [56, 39]. Perceived barriers to physical activity behaviours are an important construct in major health behaviour theories and models, including social cognitive theory [3], the theory of planned behaviour [1], the transtheoretical model [38], and the health belief model [25]. 
According to the Eurobarometer survey, the main reason for not engaging in sports or physical activity in EU Member States is a shortage of time (42\%) [19]. Other factors included the lack of motivation or interest $(20 \%)$, having a disability or illness $(13 \%)$, or that it is too expensive $(10 \%)$ [19]. These findings are supported by several national studies comprising the whole population of one country and examining the perceiving barriers to physical activity. In Australia, physically inactive adults reported lack of time (50\%), lack of enjoyment (43.9\%), and preferring to do other things (42.9\%) as the main obstacles to being physically active [24]. Finnish adults also outlined lack of time (55.6\% in men; $57.5 \%$ in women) as the main barrier, followed by a lack of motivation ( $25.6 \%$ in men; $21.6 \%$ in women), lack of company to be active with (15.9\% in men; $18.8 \%$ in women), and high expenses (9.4\% in men; $9.6 \%$ in women) [7]. A previously conducted study among Estonian adults outlined insufficient interest or will (44\%), lack of time (40\%), tiredness after work (36\%), and the excessive cost of sports $(14 \%)$ as the main perceived barriers [41]. However, the earliest studies in Estonia did not reveal the perception of perceived barriers of being physically active by different population groups. Therefore, the novelty of the current study is that it is the first to identify the perceived barriers of leisuretime physical activity according to sex, education, and age.

The aims of this study are to:

1. Describe the changes in LTPA levels among Estonian adult population during a two-year period.

2. Examine the relationships between LTPA level and SES and health indicators.

3. Explore the perceived barriers to physical activity according to different age, gender and educational groups.

The study results add important knowledge and help to plan interventions for increasing the physical activity of the population, which is stated in the document The Fundamentals of the Estonian sports policy until 2030 [18].

\section{MATERIALS AND METHODS}

\section{Subjects}

The target groups of the studies consisted of participants aged 15-69 whose permanent place of residence was in Estonia. The sample of the study was put together based on the population data of the year of conducting the study on 1 January (according to Statistics Estonia) with 1,000 persons deemed an appropriate size for a representative sample. 1009 participants took part in the survey of 2013 and 1004 respondents in the survey of 2015. 


\section{Procedures}

Cross-sectional Estonian population-based data were collected in two waves. The first study was conducted in the periods of 28/08-4/09 and $11-18 / 09 / 2013$ and was commissioned by the Estonian Olympic Committee. The second study was conducted in 14/09-4/10/2015 and was commissioned by the Estonian Ministry of Culture. Both studies were conducted by the market research company TNS EMOR. To find respondents, a proportional model of the general population was used in which all residents had an equal chance of being included in the sample. The socio-demographic structure of the sample, which formed in the course of surveying, was compared to the respective indicators of the general population by gender, age, nationality, and place of residence, and, in order to ensure representativeness, the sample was weighted to achieve compatibility with the distribution of the general population. Tablet-Assisted Personal Interviews (TAPI) were conducted by the data collectors of TNS EMOR at the respondents' homes after they had agreed to participate.

Table 1 summarises the socio-demographic characteristics of respondents by gender in both survey waves, 2013 and 2015. On the whole, the sample compositions were similar in 2013 and 2015 surveys. However, based on Pearson's chi-squared test, in 2013 there were statistically significant gender differences regarding education $(\mathrm{p}=0.000)$ and body mass index $(\mathrm{p}=0.034)$ and in 2015 regarding age $(\mathrm{p}=0.002)$ and education $(\mathrm{p}=0.000)$. Between surveys, when comparing 2013 and 2015 samples, statistical differences were evident in the case of age $(\mathrm{p}=0.000)$ and gender $(\mathrm{p}=0.000)$.

Table 1. Socio-demographic characteristics of the respondents by gender in 2013 and 2015.

\begin{tabular}{|c|c|c|c|c|c|c|c|c|}
\hline & \multicolumn{4}{|c|}{2013} & \multicolumn{4}{|c|}{2015} \\
\hline & \multicolumn{2}{|c|}{ Men } & \multicolumn{2}{|c|}{ Women } & \multicolumn{2}{|c|}{ Men } & \multicolumn{2}{|c|}{ Women } \\
\hline & $\%$ & (n) & $\%$ & (n) & $\%$ & (n) & $\%$ & (n) \\
\hline \multicolumn{9}{|l|}{ Age $\mathrm{e}^{* * *, \$}$} \\
\hline $15-24$ & 16.9 & (81) & 14.3 & (76) & 18.5 & (78) & 14.1 & $(82)$ \\
\hline $25-34$ & 20.0 & (96) & 17.4 & (92) & 22.3 & (94) & 17.5 & (102) \\
\hline $35-49$ & 28.0 & (134) & 25.7 & (136) & 30.8 & (130) & 27.7 & (161) \\
\hline $50-64$ & 25.1 & (120) & 27.7 & (147) & 21.6 & $(91)$ & 29.9 & (174) \\
\hline $65-74$ & 10.0 & $(48)$ & 14.9 & (79) & 6.9 & (29) & 10.8 & (63) \\
\hline
\end{tabular}




\begin{tabular}{|c|c|c|c|c|c|c|c|c|}
\hline & \multicolumn{4}{|c|}{2013} & \multicolumn{4}{|c|}{2015} \\
\hline & \multicolumn{2}{|c|}{ Men } & \multicolumn{2}{|c|}{ Women } & \multicolumn{2}{|c|}{ Men } & \multicolumn{2}{|c|}{ Women } \\
\hline & $\%$ & (n) & $\%$ & (n) & $\%$ & (n) & $\%$ & (n) \\
\hline \multicolumn{9}{|l|}{ Ethnicity } \\
\hline Estonian & 70.4 & (338) & 67.2 & (356) & 73.0 & (308) & 72.3 & (421) \\
\hline Other & 29.6 & (142) & 32.8 & (174) & 27.0 & (114) & 27.7 & (161) \\
\hline \multicolumn{9}{|l|}{ Education \#, \$ } \\
\hline Primary or basic & 20.4 & (98) & 13.0 & (69) & 23.5 & (99) & 15.5 & $(90)$ \\
\hline Secondary & 59.2 & (284) & 58.3 & (309) & 56.6 & (239) & 55.2 & (321) \\
\hline Higher & 20.4 & (98) & 28.7 & $(152)$ & 19.9 & $(84)$ & 29.4 & (171) \\
\hline \multicolumn{9}{|l|}{ Body mass index \# } \\
\hline $\begin{array}{l}\text { Underweight or } \\
\text { normal weight }\end{array}$ & 46.1 & (221) & 52.8 & $(280)$ & 52.1 & (220) & 48.8 & (284) \\
\hline $\begin{array}{l}\text { Overweight or } \\
\text { Obese }\end{array}$ & 53.9 & (258) & 47.2 & $(250)$ & 47.9 & (202) & 51.2 & (298) \\
\hline \multicolumn{9}{|l|}{ Satisfaction with health } \\
\hline $\begin{array}{l}\text { Completely or } \\
\text { mostly satisfied }\end{array}$ & 75.9 & (363) & 74.8 & (395) & 79.6 & (335) & 78.7 & (458) \\
\hline $\begin{array}{l}\text { Mostly not or completely } \\
\text { not satisfied }\end{array}$ & 24.1 & (115) & 25.2 & (133) & 20.4 & $(86)$ & 21.3 & (124) \\
\hline
\end{tabular}

$\mathrm{n}=$ number of respondents;

gender difference between surveys: ${ }^{* * *}$ significant at level $p<0.01 ;{ }^{* *}$ significant at level $p<0.05$; * significant at level $p<0.10$; gender differences within a survey: ${ }^{*}$ in 2013, ${ }^{\text {s }}$ in 2015.

\section{Measurements}

Physical activity level. To collect data about physical activity, a questionnaire developed by the sports sociology laboratory of the University of Tallinn in 2003 was used. The same questionnaire was also used in previous nationwide surveys in Estonia conducted in 2003, 2004, and 2006 [2, 16, 41].

LTPA was defined as regularly doing physical exercises for at least 30 minper day. The respondents were asked: "How often do you practice active hobbies, such as running, cycling, aerobics, ball games, working out in a gym, or other such activities at least $30 \mathrm{~min}$ at a time?" The answers to choose from included: a) at least four times per week; b) 2-3 times per week; c) on average, once per week; d) a few times per month; e) less frequently; f) not at all. For the analysis a) to d) were coded as physically active and e) to f) as inactive. 
Health indicators. The following question was asked to assess the health condition of the respondents: "How satisfied are you with your health in general?" The respondents were required to choose one of the following answers: a) completely; b) mostly; c) mostly not; d) not at all; e) I am not sure. For the analysis, answers were recoded as follows: a) and b) as satisfied; c) and d) not satisfied; those who were unsure were excluded from the analysis (two respondents in 2013 and one respondent in 2015).

The participants were asked to report their body height and weight in order to calculate their body mass index (BMI, $\mathrm{kg} / \mathrm{m}^{2}$ ).

Perceived barriers. The respondents who said that they practiced in LTPA on average once per week or less were asked by the interviewer to explain which factors prevented them from practicing physical hobbies: "I will read out various reasons which may prevent people from regularly practicing physical hobbies. Please state to what extent each reason applies to you." a) lack of time; b) lack of a place to play sports (hall, court, etc.); c) poor physical condition; d) I am not sufficiently interested or cannot be bothered; e) playing sports is too expensive; e) I do not have the company or people to train with; g) no means of transport for travelling for the location of playing sports; h) insufficient skills to play sports; i) tiredness after work; j) other. The respondents were asked to respond to the statements by using a threelevel scale: a) this, above all; b) this, too; c) not this reason. In the analysis, the first two categories ( $a$ and $b$ ) were coded together.

\section{Statistical analysis}

Statistical analyses were carried out with Statistical Package for the Social Sciences - SPSS, V22.0 (Chicago, IL, USA). The main descriptions of physical activity level and perceived barriers to exercise in 2013 and 2015 were presented in cross-tabulations. In addition, a chi-square $\left(\chi^{2}\right)$ statistics was used to investigate whether distributions of categorical variables differ from one another. However, in cases of statistically significant difference, chisquare does not indicate between which categories the difference lies exactly. The interrelation between physical activity (dependent variable) and sociodemographic characteristics, health-related variables, and barriers to exercise were studied in binary logistic regression models. The regression results were presented as odds ratios, which indicate the odds of the observed group (physically inactive) relative to the odds of the reference group (active) in their level of physical activity. In the dependent variable, those who are not physically active were defined as respondents who exercise less than a couple of times per month or not at all; and physically active are respondents who exercise from a couple of times per month up to four times per week. The 
odds ratio of 1 shows that the odds for being physically active are equal, while the odds above 1 indicate by how many times the odds of the observed group are higher than the odds of the reference group, and vice versa if the odds ratio is below 1 .

\section{RESULTS}

When comparing physical activity in two survey waves (2013 and 2015), it appeared that in Estonia the proportion of physically active adults has statistically significantly increased $\left(\chi^{2}=10.12\right.$, df $\left.(3), p=0.020\right)$ - in $201339 \%$ of them exercised two to three times per week or more, while in 2015 this proportion had increased to $45 \%$ (Figure 1). At the same time, the proportion of adults who were active once per week or a couple of times per month and less often or not at all active decreased by three percentage points in both groups.

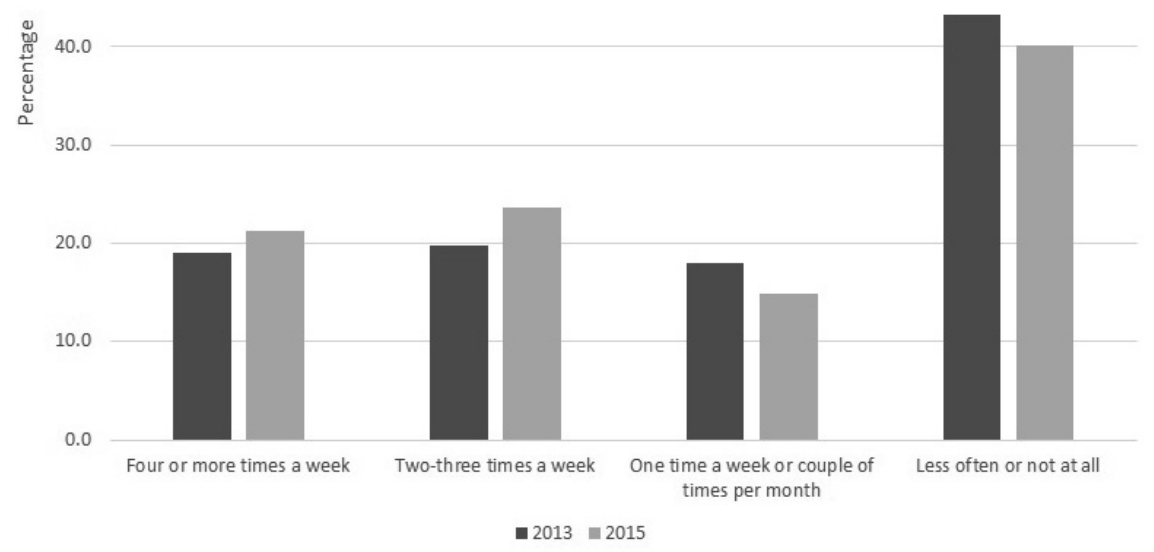

Figure 1. Leisure-time physical activity of Estonian adults in 2013 and 2015 ( \%; $n=2013$ ).

Socio-demographic and health-related characteristics juxtaposed with the likelihood of being physically inactive are presented in Table 2. One of the strongest determinants in both years was age. The analysis showed that being in the older age group increased the odds for physical inactivity compared to the youngest age group (15-24 years) $(\mathrm{p}=0.001)$ (Table 2). Although the odds ratios for age groups were somewhat lower in 2015 than 2013, still in 2015 the 25-34-year-olds had two times greater odds of being physically inactive compared to the youngest age group, and among those older than 50 , it was more than four times greater. 
Education is another significant predictor of physical inactivity - having primary or basic education increased the odds of being inactive more than two times and having secondary education more than three times compared to respondents with higher education $(\mathrm{p}=0.000)$. As for ethnicity, there was no statistically significant difference in physical activity in 2015 ( $\mathrm{p}=0.752$ ). Satisfaction with health had a statistically significant effect on the odds of being physically inactive $(\mathrm{p}=0.000)$ - in 2015 those who were not satisfied with their health had 2.5 times higher odds of being physically inactive.

Gender and body mass index showed no correlation with one's physical activity.

Table 2. The odds of being physically inactive during leisure-time physical activity among adult population in 2013 and 2015.

\begin{tabular}{|c|c|c|c|c|}
\hline \multirow{2}{*}{ Predictor } & \multicolumn{2}{|c|}{2013} & \multicolumn{2}{|c|}{2015} \\
\hline & OR & $95 \% \mathrm{Cl}$ & OR & $95 \% \mathrm{Cl}$ \\
\hline \multicolumn{5}{|l|}{ Gender (ref Female) } \\
\hline Male & 1.22 & $0.92-1.61$ & 1.10 & $0.83-1.56$ \\
\hline \multicolumn{5}{|l|}{ Age (ref 15-24) } \\
\hline $25-34$ & $2.49^{* * *}$ & $1.45-4.26$ & $2.07^{* * *}$ & $1.22-3.54$ \\
\hline $35-49$ & $3.07^{* * *}$ & $1.86-5.10$ & $3.51^{* * *}$ & $2.10-5.85$ \\
\hline $50-64$ & $5.57^{* * *}$ & $3.32-9.33$ & $4.14^{* * *}$ & $2.45-7.02$ \\
\hline $65-74$ & $7.53^{* * *}$ & $4.17-13.60$ & $4.74^{* * *}$ & $2.51-8.93$ \\
\hline \multicolumn{5}{|l|}{ Education (ref Higher) } \\
\hline Primary or basic & $2.48^{* * *}$ & $1.75-3.50$ & $2.33^{* * *}$ & $1.65-3.30$ \\
\hline Secondary & $3.84^{* * *}$ & $2.35-6.28$ & $3.14^{* * *}$ & $1.95-5.07$ \\
\hline \multicolumn{5}{|l|}{ Ethnicity (ref Estonian) } \\
\hline Other & $1.81^{* * *}$ & $1.34-2.43$ & 1.05 & $0.77-1.43$ \\
\hline \multicolumn{5}{|c|}{ Satisfaction with health (ref Satisfied) } \\
\hline Not satisfied & $1.52^{* *}$ & $1.09-2.12$ & $2.51 * * *$ & $1.78-3.53$ \\
\hline \multicolumn{5}{|c|}{ Body mass index group (ref Underweight or normal) } \\
\hline Overweight or obese & 1.11 & $0.83-1.48$ & 1.25 & $0.94-1.66$ \\
\hline Constant & $0.07^{* * *}$ & & $0.08^{* * *}$ & \\
\hline Pseudo R-Square, Nagelkerke & 0.21 & & 0.18 & \\
\hline $\mathrm{N}$ & 1,005 & & 1,003 & \\
\hline
\end{tabular}

***significant at level $p<0.01 ;{ }^{* *}$ significant at level $p<0.05$. 
Figure 2 shows barriers to exercising as perceived by those who are physically inactive. In 2015, almost all barriers were less frequently mentioned than in 2013, with the exception of feeling tired after work (respectively $55 \%$ and $48 \% ; \mathrm{p}=0.003$ ). The most frequently reported barrier for not being physically active was the absence of interest and unwillingness to make an effort (in both years more than 55\%). This was followed by tiredness after work and lack of time.

Between the two survey years, a significant decrease was present in three barriers: 1) sports being too expensive ( $\mathrm{p}=0.009), 2)$ lack of sports venues $(\mathrm{p}=0.007)$ and 3 ) lack of transportation to reach training locations $(\mathrm{p}=0.000)$. A decreasing trend $(\mathrm{p}<0.1)$ was also present for poor health $(\mathrm{p}=0.084)$ and not enough sporting skills ( $\mathrm{p}=0.072$ ).

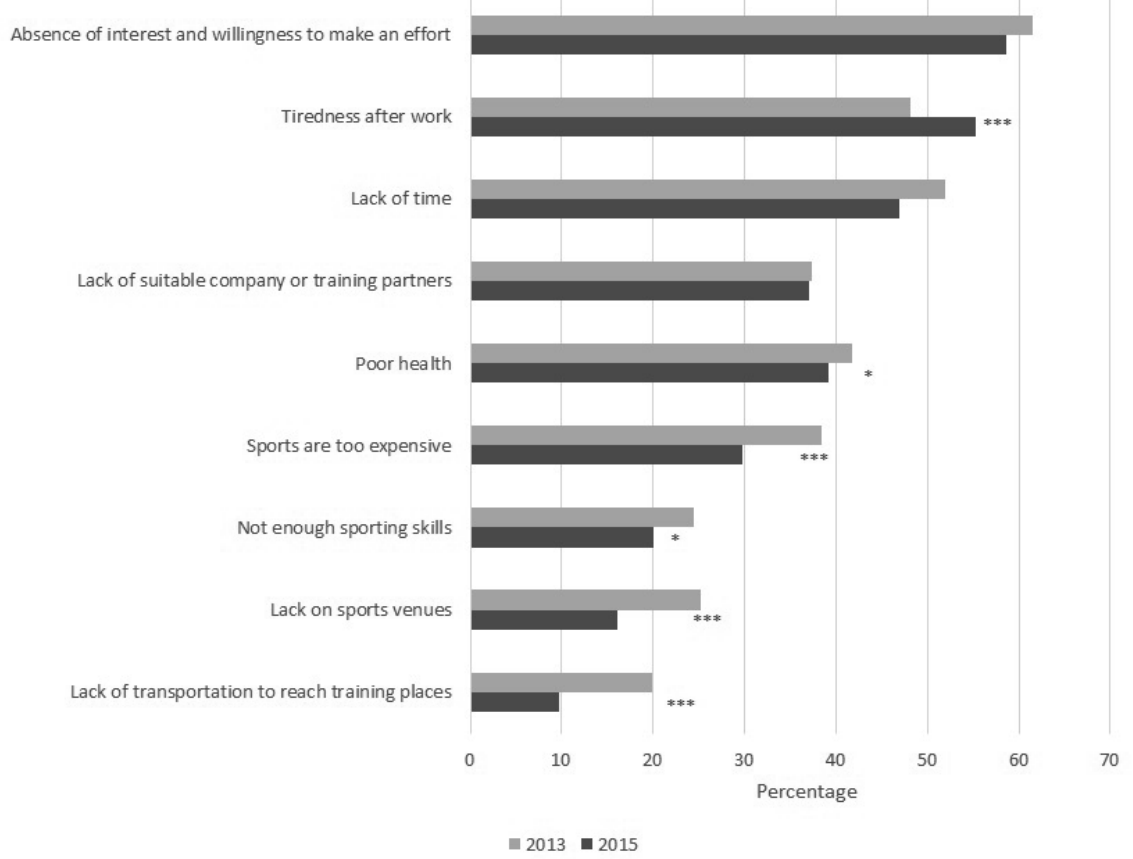

Figure 2. Barriers to being physically active as perceived by inactive survey respondents in 2013 and 2015 (\%; n=840).

${ }^{* * *}$ significant at level $p<0.01$; significant at level $p<0.1$.

Next, when looking at the differences in perceived barriers of inactive adults by gender, age, and education level, only 2015 data was used. As for gender, there were no statistically significant differences present in any of the perceived barriers (Figure 3a). As for age groups, a similarity in most frequently 
reported barriers were detected for the 15-34 and 35-49 age groups (Figure $3 b)$. The main reported barriers for these age groups were the absence of interest or unwillingness to make an effort, tiredness at work, and lack of time. Among 50-74-year-olds, the main barriers to inactivity were absence of interest or unwillingness to make an effort, poor health, and tiredness after work. A significant difference between age groups was present for tiredness at work, poor health, and lack of time ( $\mathrm{p}=0.004, \mathrm{p}=0.000$, and $\mathrm{p}=0.000$, respectively). About two-fifths of younger respondents (15-34-year-olds) stated that they did not exercise because sports are too expensive, whereas in the older age groups this is mentioned as a barrier by one-third or less $(\mathrm{p}=0.023)$. In addition, approximately $20 \%$ of respondents from the younger age groups (15-34 and 35-49) claimed a lack of sports venues as one of the barriers to physical activity, while in older age groups this is problematic for about one-tenth of respondents $(\mathrm{p}=0.012)$. As for educational level (Figure $3 c$ ), we identified two barriers which were differently perceived as a barrier depending on the educational level. More than $18 \%$ of adults with primary or basic and secondary education reported lack of sports venues as a barrier to physical activity, while only $6 \%$ with a higher education reported this $(\mathrm{p}=0.042)$. There was a tendency for those with primary or basic education and secondary education to admit poor health as a barrier to physical activity more frequently (more than $40 \%$ of respondents), whereas among those with higher education, about one-quarter felt the same $(\mathrm{p}=0.052)$.

a)

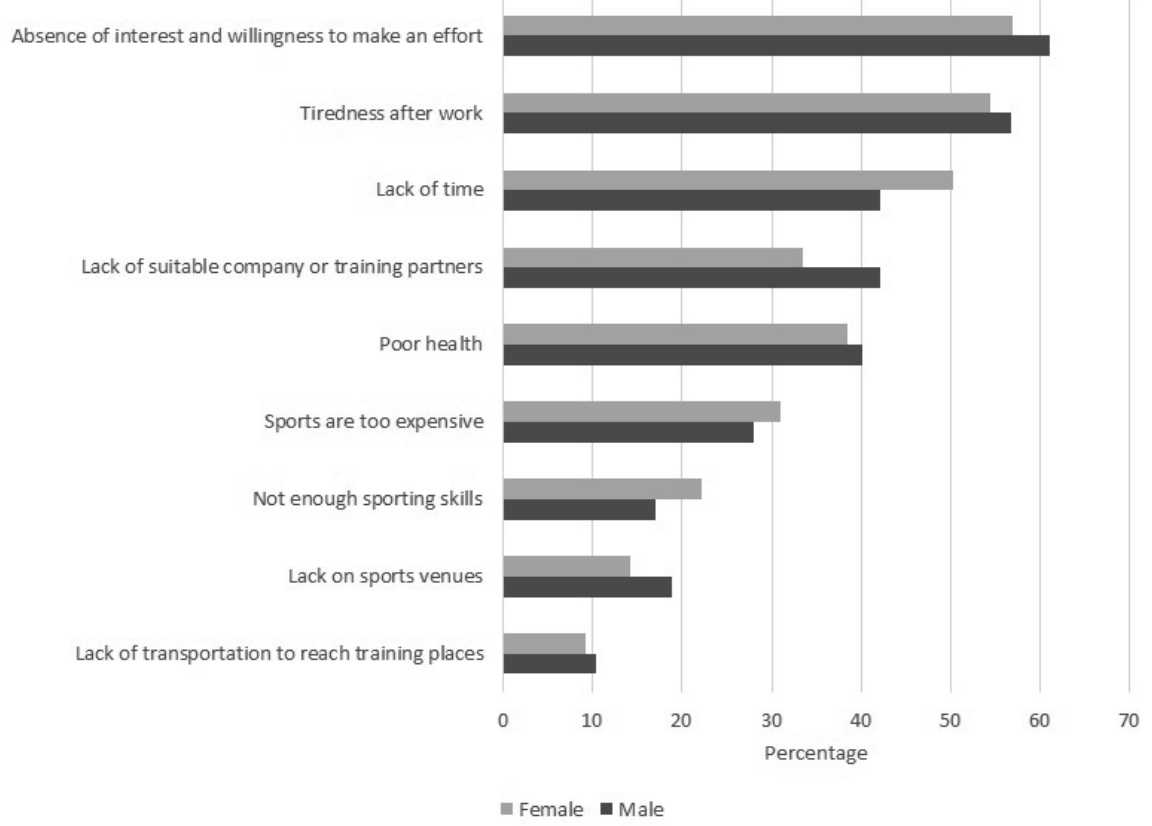


b) Absence of interest and willingness to make an effort

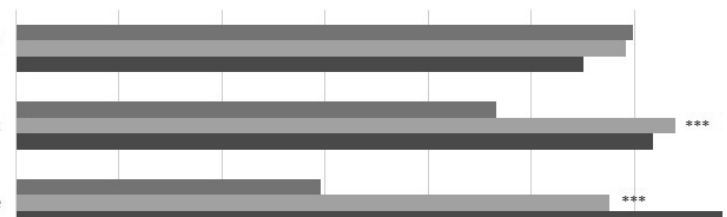

Lack of suitable company or training partners Lack of time

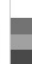

Poor health Sports are too expensive Not enough sporting skills Lack on sports venues Lack of transportation to reach training places

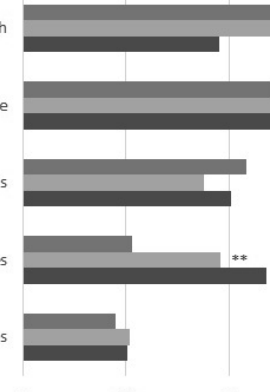
10

c)

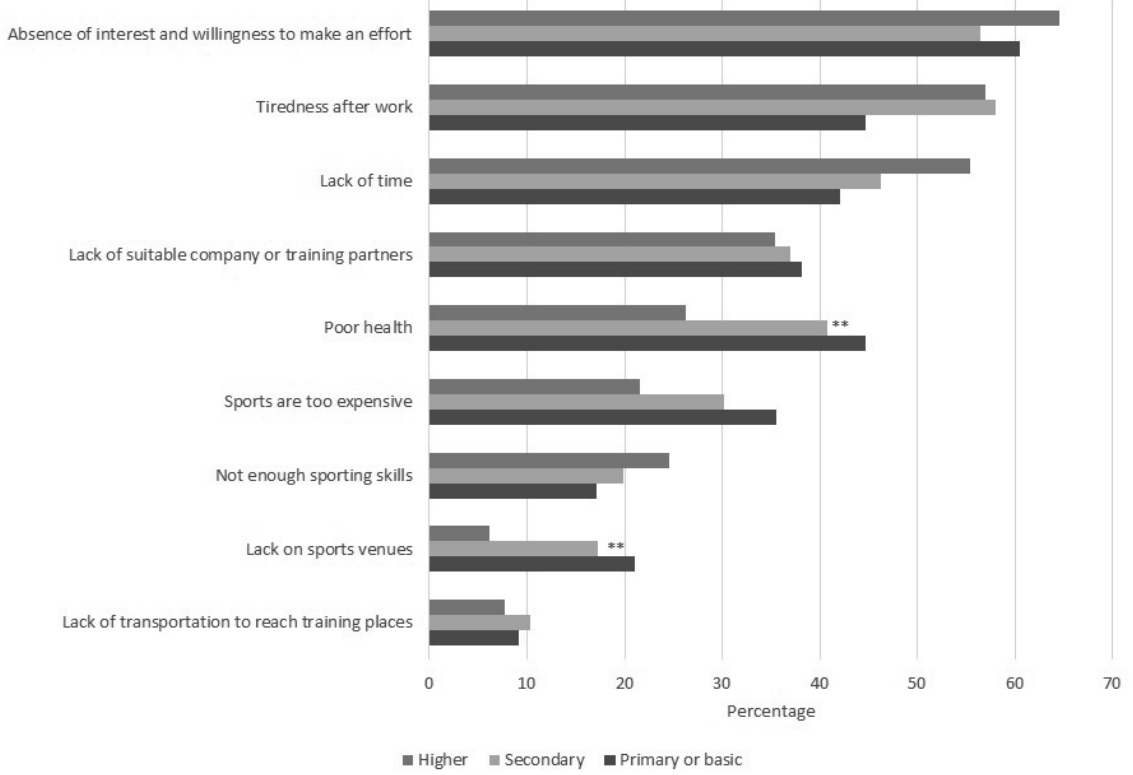

Figure 3. Barriers to being physically active perceived by inactive by gender (a), age group, (b) and educational level (c) in 2015 (\%; $n=403)$.

${ }^{* * *}$ significant at level $p<0.01 ;{ }^{* *}$ significant at level $p<0.05$. 


\section{DISCUSSION}

The aim of the current study was to describe the physical activity of Estonian adults, examine the determinants of physical activity, and also the perceived barriers. The novelty of the current study is that it is the first to identify the perceived barriers of LTPA according to sex, education, and age among Estonian inactive adults.

The overall LTPA levels of the Estonian adult population have increased modestly over the two-year period - the prevalence of adults exercising regularly at least four times per week increased from 19\% in 2013 to $21 \%$ in 2015, and those who exercise 2-3 times per week increased from $20 \%$ in 2013 to $24 \%$ in 2015. An increase in the LTPA of adults over time has also been observed in other countries. For example, in Finland the high level of LTPA increased from 1982 to 2012 by more than 12 percentage points [6], whereas in Australia the prevalence of adults acquiring at least 150 min of PA a week increased from 2002 to 2012 by more than 5 percentage points, resulting in a prevalence of $61 \%$ of adults being sufficiently physically active [13], which is three times greater than the current study. The systematic review by Knuth and Hallal [28] also confirmed an increasing trend in the leisure time physical activity of adults. However, there are still populations where a decline in LTPA $[28,30]$ or relatively stable LTPA levels has been observed [8]. At the same time, it has been highlighted that despite the slight increase in LTPA, due to declining work-related activity, declining transportation activity, declining activity in the home, and increasing sedentary activity, the total physical activity levels are declining [8]. It should be pointed out that despite the modest increase in LTPA of Estonian adults in the current study, the physical activity levels are worrying as less than half of the adults exercise more than once per week during their leisure time, which is below the average of the $28 \mathrm{EU}$ member states [19].

Physical activity is a dynamic process throughout a person's lifespan. Trost et al. [52] have concluded in their review that age and gender are the two most consistent demographic correlates of physical activity in adults. As for age, our results are in accordance with previous studies as the odds of being physically inactive increased with age $[5,43,52,53]$. This indicates that there is a need to pay special attention to supporting the physical activity levels of the older age group. Public health and sport policy documents should include actions related to physical activity opportunities for older adults, especially when the general trend in the population demographic situation is an ageing population. However, up to now there has not been any health promotion campaigns in Estonia targeted at older adults [15]. 
Therefore, our results highlight a need to create additional interventions and solutions supporting physical activity in people from the older age group.

When considering the physical activity levels from the perspective of gender, several studies have found that men are more physically active than women $[5,29,52,22]$. Surprisingly, this trend was not supported in the current study where no gender differences were present between active and inactive adults. What is even more important is the fact that among inactive adults, there were no gender differences present in perceived barriers. We can hypothesise that existing sports facilities, available forms of LTPA, and existing gender roles in Estonian society support physical behaviour regardless of gender. Another possible explanation for why this study does not support the results of previous studies can arise from how physical activity is measured and what criteria are used to defining people as physically active or inactive. For example, Hamrik et al. [21] divided adults into three activity groups (high-, moderate-, and low-active) based on their weekly MET-min and showed that while there were no gender differences in the probability of being low-active, gender differences were present for high-active adults.

Another demographic factor related to the physical activity of adults is educational level. There is a consensus that a higher educational level is associated with higher levels of physical activity $[5,43,52]$ and our results are in line with previous studies. As for perceived barriers, we identified only two barriers that were differently perceived by adults depending on their educational level. One of these barriers was the lack of sports venues: only $6 \%$ of inactive adults with a higher education reported it as a barrier to physical activity compared to $18 \%$ of adults with a secondary, primary, or basic educational level. It could be hypothesised that persons with a higher education have better access to sporting venues as their presumably better economic status enables a wider range of choices. At the same time, as a considerable number of adults with basic or primary education are young people, it also could be that there are not enough sporting facilities available for playing the sports that they enjoy (e.g. extreme sports). Another barrier where differences based on educational level were present, was poor health: those with a higher education reported it less often as a barrier than adults with lower educational levels. The better health of adults with a higher education could be related to their presumably better knowledge and health behaviour and is also supported by the overall tendency that higher educational level is associated with lower rates of incidence and morbidity [20,57].

It has been concluded that health status is one of the determinants of physical activity [5]. This tendency is also supported by the current study where those who were not satisfied with their health had 2.5 times greater 
odds of being physically inactive. At the same time, it is well-known that physical activity is an important factor for preventing and controlling several diseases [36]. We can argue that for those who are not satisfied with their health, a family physician can play an important role in supporting and motivating them to do health-enhancing physical activity. As people with more health problems tend to visit the family physician more often, this puts the physician in a favourable position to give exercise-related advice. It has been shown that getting physical activity-related advice from a physician significantly increases the physical activity levels of older adults [26]. Also, according to a meta-analysis, the promotion of physical activity among sedentary adults by a primary care physician leads to a small to medium improvement in self-reported physical activity after twelve months [34]. In Estonia, according to the physicians' reports, the most important topic in counselling for health promotion was physical activity [48]. However, the effect of the counselling and how the current system should be supported and improved warrants further research.

The most prevalent barriers to physical activity reported by inactive adults were 1) the absence of interest or unwillingness to make an effort, 2) tiredness at work, and 3) lack of time, which are common barriers reported by other studies [7, 24, 30, 52]. However, the proportion of adults reporting these barriers is more than two times higher in the current study than in the study conducted in Poland [30]. When comparing the two measurement periods in the current study, it is alarming that workrelated tiredness as a barrier to physical activity has increased by seven percentage points. According to the previous study, high work stress and work demand have a negative impact on LTPA [27]. Thus, it is important to raise the knowledge of employers concerning the positive influence of physical activity on health. A meta-analysis by Conn et al. [12] suggested that workplace physical activity interventions not only increase overall physical activity but also improve work attendance, job satisfaction, and reduce job stress. Of course, supporting the physical activity of employees is not only a task for employers, but can also be supported by the state through legislation supporting physical activity among both employers and employees.

One important and positive finding of the current study was that during the two-year period, significant changes were present for several environmental factors - there were significantly fewer inactive adults reporting lack of sports venues, lack of transportation to training places, and the expense of sporting as a barrier to being physically active. In addition, among inactive adults, no differences could be noted between men and women regarding 
environmental determinants of physical activity which is in line with previous findings [55].

One limitation of the study is the self-reported measurements of physical activity, which did not allow for a precise quantification of the intensity and duration of physical activity. Moreover, a recall bias could have affected the reported physical activity levels. However, using more precise objective methods in a similar sample size is rarely used and would need significantly greater resources. Another limitation is that perceived barriers were only asked from inactive adults which did not enable a comparison between barriers in active and inactive adults. However, this is the first study in Estonia examining the gender, educational, and age differences in perceived barriers of inactive adults, thus providing important input into public health strategies.

In conclusion, over the two-year period positive changes in the LTPA of adults can be noticed. However, the physical activity levels are still rather low and the odds of being inactive are greater in the older age group. As the perceived barriers among inactive adults are different depending on the age group, public health strategies should consider age-specific solutions. Also, the high prevalence of adults reporting lack of time and tiredness after work as a barrier to physical activity indicates that all sectors outside the health sector should be involved in supporting the physical activity levels of adults.

\section{REFERENCES}

1. Ajzen I. (1991) The theory of planned behavior. Organ Behav Hum Dec, 50: 179-211

2. Arvisto M, Undusk R, Paju K, Truu E, Noormets, J. (2004) Physical activity and its perspectives among Estonian adult population. Survey, Tallinn

3. Bandura A. (1986) Social foundations of thought and action: A social cognitive theory. Englewood Cliffs, NJ: Prentice Hall

4. Bauman AE. (2004) Updating the evidence that physical activity is good for health: an epidemiological review 2000-2003. J Sci Med Sport, 7: 6-19

5. Bauman, AE, Reis RS, Sallis JF, Wells JC, Loos RJ, Martin BW. (2012) Correlates of physical activity: why are some people physically active and others not? The Lancet, 380: 258-271

6. Borodulin K, Harald K, Jousilahti P, Laatikainen T, Männistö S, Vartiainen E. (2016) Time trends in physical activity from 1982 to 2012 in Finland. Scand J Med Sci Sport 26: 93-100

7. Borodulin K, Sipilä N, Rahkonen O, Leino-Arjas P, Kestilä L, Jousilahti P. (2016) Socio-demographic and behavioral variation in barriers to leisure-time physical activity. Scand J Public Health 44: 62-69 
8. Brownson RC, Boehmer TK, Luke DA. (2004) Declining rates of physical activity in the United States: What are the contributors? Ann Rev Public Health, 26: 421-443

9. Caspersen CJ, Powell KE, Christensen GM. (1985) Physical activity, exercise, and physical fitness: definitions and distinctions for health-related research. Public Health Reports, 100: 126-131

10. Cavill N, Kahlmeier S, Racioppi, F (2006) Physical activity and health in Europe. Evidence for Action. WHO

11. Chief Medical Officer. (2004) At least five a week: evidence on the impact of physical activity and its relationship to health: a report from the Chief Medical Officer. London: Department of Health

12. Conn VS, Hafdahl AR, Cooper PS, Brown LM, Lusk SL. (2009) Meta-analysis of workplace physical activity interventions. Am J Prev Med, 37: 330-339

13. Devonshire-Gill KR, Norton KI. (2018) Australian adult physical activity sufficiency trend data: positive, prevalent, and persistent changes 2002-2012. J Phys Act Health, 15: 117-126

14. Eime R, Young J, Harvey J, Charity M, Payne W. (2013) A systematic review of the psychological and social benefits of participation in sport for adults: Informing development of a conceptual model of health through sport. Int J Behav Nutr Phys Act, 10: 14

15. Estonian Sport for All Association (ESFA). (2018) Annual report 2017

16. Estonian Ministry of Culture. (2003) Culture consumption among Estonian population in 2003. Survey, Tallinn

17. Estonian National Institute for the Health Development. (2005) Health behavior and health among the Estonian adult population in 2004

18. Estonian Parliament. (2015) The Fundamentals of the Estonian sports policy until 2030. RT III, 20.2.2015, 2

19. European Commission. (2014) Special Eurobarometer 412. Sport and physical activity. European Commission, Directorate-General for Education and Culture and coordinated by Directorate-General for Communication, Brussels. Retrieved January 30, 2018 from http://ec.europa.eu/health/nutrition_physical_activity/docs/ebs_412_en.pdf

20. Hahn RA, Truman BI. (2015) Education improves public health and promotes health equity. Int J of Health Serv : Planning, Administration, Evaluation, 45: $657-678$

21. Hamrik Z, Sigmundová D, Kalman M, Pavelka J, Sigmund, E. (2014) Physical activity and sedentary behaviour in Czech adults: Results from the GPAQ study. Eur J Sport Sci, 14: 193-198

22. Hartmann-Tews, I. (2006) Social stratification in sport and sport policy in the European Union. Eur J Sport Soc, 3: 109-124

23. Haskell WL, Lee IM, Pate RR, Powell, KE, Blair SN, Franklin BA, Macera CA, Heath GW, Thompson BD, Bauman A. (2007) Physical activity and public health: updated recommendation for adults from the American College of Sports Medicine and the American Heart Association. Circulation, 116: 1081-1093 
24. Hoare E, Stavreski B, Jennings GL, Kingwell BA. (2017) Exploring motivation and barriers to physical activity among active and inactive Australian adults. Sports 5: 47

25. Janz NK, Becker, MH. (1984) The Health Belief Model: A decade later. Health Educ Quart, 11: 1-47

26. Kerse N, Elley CR, Robinson E, Arroll, B. (2005) Is physical activity counseling effective for older people? A cluster randomized, controlled trial in primary care. J Am Geriatr Soc, 53: 1951-1956

27. Kirk MA, Rhodes RE. (2011) Occupation correlates of adults' participation in leisure-time physical activity: a systematic review. Am J Prev Med, 40: 476-485

28. Knuth AG, Hallal PC. (2009) Temporal trends in physical activity: a systematic review. J Phys Act Health, 6: 548-559

29. Koeneman MA, Verheijden MW, Chinapaw MJM, Hopman-Rock, M. (2011) Determinants of physical activity and exercise in healthy older adults: A systematic review. Int J of Behav Nutr Phys, 8: 142

30. Kwaśniewska, M, Pikala, M, Bielecki W, Dziankowska-Zaborszczyk E, Rębowska, E, Kozakiewicz K, Drygas W. (2016) Ten-Year changes in the prevalence and socio-demographic determinants of physical activity among Polish Adults Aged 20 to 74 Years. Results of the National Multicenter Health Surveys WOBASZ (2003-2005) and WOBASZ II (2013-2014). Plos One, 11: e0156766

31. Leslie E, Cerin E, Gore C J, St. George A, Bauman, A and Owen, N. (2004) Gender, age, and educational-attainment differences in Australian adults' participation in vigorous sporting and fitness activities. J Phys Act Health, 1:377-388

32. Lusmägi P, Einasto M, Roosmaa E-L. (2016) Leisure-time physical activity among different social groups of Estonia: Results of the national physical activity survey. Phys Cult Sport Stud Res, 69: 43-52

33. Nunan, D, Mahtami K, Roberts N, Heneghan C. (2013) Physical activity for the prevention and treatment of major chronic disease: An overview of systematic reviews. Systematic Reviews, 2, 56

34. Orrow G, Kinmonth A-L, Sanderson S, Sutton S. (2012) Effectiveness of physical activity promotion based in primary care: systematic review and meta-analysis of randomised controlled trials. BMJ, 344: e1389

35. Patel KV, Coppin AK, Manini TM, Lauretani F, Bandinelli S. (2006) Midlife physical activity and mobility in older age The InCHIANTI Study. Am J Prev Med, 31: 217-24

36. Pedersen BK, Saltin B. (2015) Exercise as medicine - evidence for prescribing exercise as therapy in 26 different chronic diseases. Scand J Med Sci Sport, 25: $1-72$

37. Physical Activity Guidelines Advisory Committee (Ed.): Physical Activity Guidelines Advisory Committee Report 2008. Washington, DC: U.S. Department of Health and Human Services 
38. Prochaska JO, Marcus BH. (1994) The transtheoretical model: Application to exercise. In R. K. Dishman (ed.), Advances in exercise adherence. Champaign, IL: Human Kinetics, 161-180

39. Ronda G, Van Assema P, Brug J. (2001) Stages of change, psychological factors and awareness of physical activity levels in The Netherlands. Health Promot Int, 16: $305-314$

40. Ruston D, Hoare J, Henderson L. (2004) National Diet and Nutrition Survey: Adults Aged 19-64 Years, Vol. 4: Nutritional Status (Anthropometry and Blood Analytes), Blood Pressure and Physical Activity. The Stationery Office: London

41. Saar Poll. (2006) Culture Consumption among Estonian population in 2006. Survey, Tallinn

42. Sallis JF, Owen N. (1999) Physical Activity and Behavioral Medicine. Thousand Oaks, CA Sage Publications: 110-134

43. Salmon J, Owen N, Bauman A, Schmitz MKH, Booth M. (2000) Leisure-Time, occupational, and household physical activity among professional, skilled, and less-skilled workers and homemakers. Prev Med, 30: 191-199

44. Scheerder J, Vandermeerschen, H, Van Tuyckom C, Hoekman R, Breedveld K, Vos S. (2011) Understanding the game: sport participation in Europe: facts, reflections and recommendations. KU Leuven/Research Unit of Social Kinesiology and Sport Management

45. Seo, D-C, Torabi, M. (2007) Differences in vigorous and moderate physical activity by gender, race/ethnicity, age, education, and income among U.S. Adults. AM J Health Educ, 38: 122-128

46. Sharpe PA, Granner ML, Hutto B et al. (2004) Association of environmental factors to meeting physical activity recommendations in two South Carolina counties. Am J Health Promot, 18: 251-257

47. Stamatakis E, Hirani V, Rennie K. (2009) Moderate-to-vigorous physical activity and sedentary behaviours in relation to body mass index-defined and waist circumference-defined obesity. Brit J Nutr 101: 765-773

48. Suija K, Pechter Ü, Maaroos J, Kalda R, Rätsep A, Oona M, Maaroos HI. (2011) Arstide kehaline aktiivsus ja nõuannete jagamine oma patsientidele liikumisharjumuse kujundamise kohta. Eesti Arst, 90: 116-121

49. The Health and Social Care Information Centre. (2009) Health Survey for England 2008. Retrieved January 31, 2018 from https://digital.nhs.uk/catalogue/PUB00430

50. Tekkel M, Veideman T. (2015) Health Behaviour among Estonian Adult Population, 2016. Estonian National Institute for Health Development. Survey, Tallinn

51. Tekkel M, Veideman T. (2017) Health Behaviour among Estonian Adult Population, 2016. Estonian National Institute for Health Development. Survey, Tallinn

52. Trost SG, Owen N, Bauman AE, Sallis JF, Brown W. (2002) Correlates of adults participation in physical activity: Review and update. Med Sci Sport Exer 34: 1996-2001 
53. van Stralen MM, De Vries H, Mudde AN, Bolman C, Lechner L. (2009) Determinants of initiation and maintenance of physical activity among older adults: a literature review. Health Psychol Rev, 3: 147-207

54. Van Tuyckom C, Scheerder, J. (2010) Sport for All? Insight into stratification and compensation mechanisms of sporting activity in the EU-27. Sport Educ Soc 15: 495-512

55. Wendel-Vos W, Droomers M, Kremers S, Brug J, Van Lenthe F. (2007) Potential environmental determinants of physical activity in adults: a systematic review. Obes Rev, 8: 425-440

56. Wilcox S, Richter DL, Henderson KA, Greaney ML, Ainsworth BE. (2002) Perceptions of physical activity and perceived barriers and enables in African American women. Ethnic Dis, 12: 353-362

57. Winkleby MA, Jatulis DE, Frank E, Fortmann SP. (1992) Socioeconomic status and health: how education, income, and occupation contribute to risk factors for cardiovascular disease. Am J Public Health, 82: 816-820

58. World Health Organization. (2009) Global Health Risks: Mortality and Burden of Disease Attributable to Selected Major Risks

59. World Health Organization. (2014) Global recommendations on physical activity for health

\section{Correspondence to:}

Peeter Lusmägi

Institute for International Social Studies,

School of Governance, Law and Society

Tallinn University, Estonia

E-mail: peeter@eok.ee 